\title{
Protection Against Parkinson's Disease Progression: Clinical Experience
}

\author{
Peter A. LeWitt* ${ }^{\dagger}$ and Danette C. Taylor** \\ *Department of Neurology, Henry Ford Hospital, Detroit, Michigan; ${ }^{\dagger}$ Department of Neurology, Wayne State University School \\ of Medicine, Detroit, Michigan; and ${ }^{\ddagger}$ Department of Neurology and Ophthalmology, Michigan State University School of \\ Osteopathic Medicine, East Lansing, Michigan
}

\begin{abstract}
Summary: Treatments with potential neuroprotective capability for Parkinson's disease (PD) have been investigated in randomized, controlled, clinical trials and other studies since the mid-1980s. Although promising leads have arisen, no therapy has been proven to halt or slow disease progression. Several large-scale studies have highlighted progress in methodology, as well as the frustrations of translating laboratory science to practical applications. This review summarizes findings from clinical trials with several classes of compounds, including monoamine oxidase-B inhibitors (selegiline, lazabemide, rasa-
\end{abstract}

giline), dopaminergic drugs (ropinirole, pramipexole, levodopa), antioxidant strategies ( $\alpha$-tocopherol), mitochondrial energy enhancers (coenzyme $\mathrm{Q}_{10}$, creatine), antiapoptotic agents (TCH346, minocycline, CEP-1347), and antiglutamatergic compounds (riluzole). Beyond small-molecule pharmacology, gene therapy approaches, such as delivering neurotrophic substances (e.g., neurturin) by viral vector, are the next generation of treatment options. Key Words: Parkinson's disease, neuroprotection, neurodegeneration, clinical trials, disease modification.

\section{INTRODUCTION}

Parkinson's disease (PD) is unique among the other neurodegenerative disorders because of the almost palpable anticipation of an imminent cure. So much is known about its pathophysiology that speculation among PD optimists is not if, but when, a definitive treatment will arrive. Multiple examples of the translation of preclinical science to clinical research have provided evidence of this enthusiasm. The number and variety of completed clinical trials for PD neuroprotection has exceeded those conducted for all other neurodegenerative disorders. Explaining this productivity is the intense interest that PD has engendered in the neuroscience community.

Clues derived from study of the PD brain have led to an abundance of preclinical research. Since the mid1970s, thousands of research reports have investigated possible disease mechanisms and neuroprotective approaches, using a variety of animal or cellular models of

Address correspondence and reprint requests to: Peter A. LeWitt, M.D., Henry Ford Health Systems-Franklin Pointe Medical Center, 26400 West Twelve Mile Road, Suite 180, Southfield, MI 48034. E-mail: palewitt@ameritech.net.
PD. Despite the known limitations of such models, ${ }^{1,2}$ they have been extremely influential in guiding the search for pharmacological interventions that might halt disease progression. A discussion of all the diverse therapeutic approaches currently under exploration (antioxidant, pro-metabolic, antiapoptotic, antiexcitotoxic, and anti-inflammatory, among others) is beyond the scope of this report-or any review wishing to provide up-to-date information-as new research on this topic continues to appear at an accelerating pace.

In 2003, a National Institutes of Health (NIH) committee recommended 59 candidate compounds for testing as potential neuroprotective agents of $\mathrm{PD}^{3}-\mathrm{a}$ list that, only 5 years later, has greatly different options. The discussion in this review focuses on completed clinical studies and their implications for understanding the cause or causes of PD. This topic has been the subject of several reviews in recent years, including evidence-based therapeutics assessments by a subcommittee of the American Academy of Neurology ${ }^{4}$ and by a task force of the Movement Disorder Society. ${ }^{5,6}$

Symptomatic treatment of PD has obvious endpoints, including those rated by evaluation of disabilities, changes in motor features, onset of dyskinesias or motor fluctuations, and quality of life assessments. With respect 
to disease modification in PD, however, the measure of an effective treatment outcome is less clear. Underlying the design of clinical trials for neuroprotection in PD is an ongoing search for a compelling conceptual definition.

Several recent reviews have expounded on the varieties of neuroprotective agents and possible mechanisms for intervening against neuronal loss in the substantia nigra pars compacta $(\mathrm{SNpc}){ }^{7}$ which is the presumptive definition for most researchers. Neuroprotection can be defined from a clinical perspective: halting or slowing the emergence or worsening of disability in everyday activities, diminishing the decline of ratings focused on distinctive parkinsonian features, or avoidance of specific clinical milestones (such as the perceived need for starting dopaminergic therapy). The latter endpoint, despite its apparent imprecision, has proven to correlate well to more objective ratings of parkinsonism and was used as the primary endpoint in the first clinical trial of neuroprotection, the DATATOP study (Deprenyl and Tocopherol Antioxidative Therapy of Parkinsonism). ${ }^{8-10}$ The "need for initiation of dopaminergic therapy" has also served as a primary or secondary endpoint for several additional investigations. Other studies have used specific ratings of parkinsonian signs and symptoms (including timed tests or neuroimaging of the dopaminergic system in the brain) for the measure of therapeutic effectiveness.

Regardless of the clinical investigative tools used, researchers must recognize that the worsening of parkinsonian symptomatology may not be a direct reflection of what is occurring at a cellular or neurochemical level. For example, there is no consensus that neuroprotection needs to be a therapeutic intervention that would prevent the development of Lewy bodies, the pathological hallmark of PD. Furthermore, observed changes in parkinsonian features over time might have alternative explanations besides the continuing death of additional dopaminergic neurons. Changes that might appear to represent the declining number of SNpc neurons include such alternative explanations as metabolic compromise among the surviving neuronal population (such as the well-documented finding of impaired mitochondrial function ${ }^{11,12}$ ). Alterations in the structure of dopaminergic nerve terminals ${ }^{13}$ or loss of cellular viability because of impaired protein folding or turnover ${ }^{14}$ are also mechanisms that can explain worsening of parkinsonism without necessarily invoking additional neuronal death.

Another possibility for explaining the worsening of clinical symptomatology includes the gradual loss of compensatory responses in remaining SNpc neurons that, initially, were able to increase dopaminergic output. ${ }^{15}$ Progressive loss of SNpc dopaminergic neurons is, of course, a well-defined pathological outcome in PD that correlates with disease severity ${ }^{7}$; however, even this ob- vious target for a biomarker has proven to be problematic. At present, it is difficult for clinicians to link worsening in clinical state with decline in the SNpc population. The ELLDOPA study ${ }^{16}$ (a recent clinical trial described further in the section on dopaminergic drugs) exemplifies this quandary, in that a neuroimaging marker of the SNpc dopaminergic nerve terminal population provided results opposite to the clinical observations of the study.

Although the ideal neuroprotective treatment would be $100 \%$ effective, partial control of progression or prevention of various undesired outcomes of advancing PD (for example, development of dementia) need to be envisioned in conceptualizing neuroprotection. Clinical trials for neuroprotection have used various criteria for gauging a clinically meaningful outcome, but the problem of defining effective neuroprotection has more fundamental challenges.

Since the 1980s, a rating for cataloging and quantifying parkinsonian severity has gained widespread acceptance for neuroprotection studies. The Unified Parkinson Disease Rating Scale ${ }^{17}$ (UPDRS) is especially useful in this regard, because untreated PD patients tend to show near-linear decline in scores over several years. ${ }^{9,10,16,18}$ If the pattern of worsening in a particular study is not linear over time, various methods have been developed for analyzing such nonlinear changes. ${ }^{19}$

One problem posed by the UPDRS is that its score consists of summed descriptive ratings that may be relatively insensitive to small changes in clinical state. This is especially problematic for subjects with mild features of parkinsonism, as typically enrolled in a neuroprotection study. The UPDRS is currently undergoing revision, ${ }^{20}$ but the updated version will not necessarily enhance sensitivity to small changes in clinical progression. Purely quantitative methods seem to be desirable, especially those targeting slowed movements, which is often regarded as the core feature of parkinsonian disability. In the DATATOP study, a quantitative measurement of motor performance, the timed Purdue pegboard task of motor dexterity, was investigated ${ }^{8-10}$; however, such testing did not provide any clear advantage over the results of UDPRS ratings as an indicator of disease progression or disability.

Surrogate markers of PD progression have been sought as alternative to clinical rating. Neuroimaging of the dopaminergic system with a labeled levodopa analog $\left(\left[{ }^{18} \mathrm{~F}\right]\right.$ fluorodopa) with positron emission tomography (PET) or ligands of dopamine transporter uptake sites with single photon emission computed tomography (SPECT) have been incorporated into several of the clinical studies. Although these neuroimaging techniques offer scientifically compelling views of dopaminergic function in PD and can also offer near-linear changes over time, their potential susceptibility to artefactual 
changes continues to be controversial. ${ }^{21-23}$ Evaluating impairment of the motor network using metabolic maps from $\left[{ }^{18} \mathrm{~F}\right]$ fluoro-2-deoxyglucose PET is a newly developed approach to view worsening in the PD brain. ${ }^{24,25}$ Beyond imaged dopaminergic nigrostriatal pathways, the search for other indirect indicators of disease progression has not led to reliable biomarkers in the living PD patient.

One methodological approach that has been incorporated into recent PD neuroprotection research has been the so-called "futility trial". This study design, used extensively in cancer therapeutics research, offers one means for economy in the initial stages of drug exploration. ${ }^{26}$ Several recent PD neuroprotection trials have used this study methodology, including four treatments investigated in a consortium program called Neuroprotection Exploratory Trials in Parkinson's Disease (NETPD), funded and directed by the NIH National Institute of Neurological Disorders and Stroke. ${ }^{3}$ Compared with conventional clinical trials for PD treatments, a study for testing a predetermined threshold of futility is designed and powered to rule out the need for further investigation of a therapeutic intervention. If initial results indicate that such a study does not reach a statistically significant endpoint of futility to proceed further, then its alternative conclusion will be that a more extensive efficacy investigation is warranted. Given the large number of possible PD neuroprotective interventions under consideration, ${ }^{3}$ the futility trial approach offers the potential for achieving a faster pace in clinical research.

Several categories of potentially neuroprotective treatments have been investigated with pharmacological interventions. Discussion of neuroprotection studies with compounds possessing neurotrophic properties, such as GM1-ganglioside and recombinant glial cell line-derived neurotrophic factor (liatermin; Amgen, Thousand Oaks, $\mathrm{CA})$ can be found in an accompanying article. ${ }^{27}$

\section{MONOAMINE OXIDASE INHIBITORS}

\section{Selegiline (deprenyl)}

It has been more than two decades since the start of the first clinical trials to evaluate potential neuroprotective treatments. Researchers in the 1980s were especially focused on the possibility that oxidative stress contributed to the initiation and progression of PD. Oxidative stress affecting SNpc neurons was envisioned to be the most likely factor causing progressive neuronal loss. ${ }^{28}$ The selective vulnerability of dopaminergic SNpc neurons and their terminals in the striatum was hypothesized to be linked to a number of pro-oxidant factors, among them the local presence of reactive iron and melanin, which promote the generation of free radicals. ${ }^{29}$ The ability of dopamine to auto-oxidize to compounds acting as oxyradicals provided another possibility for toxicity related to this neurotransmitter. Finally, the catabolism of dopamine by monoamine oxidase (MAO) was thought to be a means for generating potentially toxic byproducts. At sites within neurons and in nearby glia, the turnover of dopamine by MAO yields the hydroxyl radical and other reactive oxygen species. ${ }^{30}$

With the intention of blocking the formation of oxyradicals resulting from dopamine metabolism, several clinical investigations targeting MAO were initiated. In each instance, the compound chosen to inhibit this pathway was selegiline (also known at the time as deprenyl), an MAO inhibitor selective for the type B isomer when administered at a dose up to $10 \mathrm{mg} /$ day. Selegiline is an irreversible (suicide-type) MAO-B inhibitor. Because MAO-B was thought to be the primary pathway for dopamine catabolism, inhibiting the other isomer of MAO (MAO type-A) did not seem to be necessary for significantly limiting dopamine turnover in the striatum and, consequently, generation of oxyradicals.

In the mid-1980s, three studies were conducted to address the possibility that selegiline was neuroprotective in PD. The largest of these, DATATOP, was initiated in 1987 and had been planned to be conducted over 2 years after the recruitment of all 800 subjects. ${ }^{8}$ This placebo-controlled, double-blind study also involved a treatment arm in which another antioxidant, $\alpha$-tocopherol, was also administered in a $2 \times 2$ factorial study design (see further under Antioxidant Strategies). For the DATATOP study, the hypothesis was that chronic selegiline would lessen oxidative stress generated from dopamine turnover. The result in the environment of the SNpc neurons presumably would be less damage and less loss of these neurons, with concomitant slowing progression of PD. Participants in the DATATOP study were subjects with mild ("early") clinical features of PD and no current need for symptomatic treatment of parkinsonism. The clinical outcomes of these pharmacological interventions were repeated outpatient assessments, at which time subjects were assessed for the need to initiate symptomatic dopaminergic therapy because of emerging disability. Subjects also underwent objective ratings of PD disability and clinical features using the UDPRS, and measurements of CSF dopamine metabolism markers were made at the start and end of the study.

Although the plan was for breaking the "blind" at 24 months, the DATATOP study protocol was altered midway in the first year because a major treatment effect was detected through an interim analysis. ${ }^{9,10}$ The selegiline treatment group, after a mean of $12 \pm 5$ months, was found to reach the endpoint of the study at significantly lower rates. The need for dopaminergic treatment occurred for 176 of 401 subjects receiving placebo, compared with 97 of 399 selegiline-treated subjects $(p<$ $10^{-8}$, as evaluated by Fisher's exact test for the proportions of subjects reaching the study endpoint). A Kaplan- 
Meier analysis for sequential occurrences of endpoints revealed that selegiline reduced the risk for needing dopaminergic treatment by approximately half of the rate with placebo. Another finding from the DATATOP study was that treatment with selegiline lowered the risk for eventually developing freezing of gait. ${ }^{31}$

This conclusion changed a few months later. Subsequent Kaplan-Meier analysis showed that the mean selegiline endpoint did not differ from that of placebo even though, earlier in the trial, there had been a robust difference. This paradox was explored in a number of ways post hoc, without a clear explanation. One possibility was that selegiline conferred a small but detectable symptomatic anti-parkinsonian effect that persisted beyond its 4 -week washout. ${ }^{32}$ To test for this possibility, the protocol was modified to include a staggered washout of selegiline to investigate the effects on the clinical state. The conclusion was evidence that selegiline continued to exert some improvement of parkinsonian features at 4 weeks and beyond. Hence, determination of neuroprotective effect may have been confounded by this mild but significant symptomatic action, the magnitude of which may have changed over time. Other issues uniquely faced by the DATATOP trial include its novel primary endpoint ("need for dopaminergic therapy") which, though correlated to UPDRS scores, ${ }^{9,10}$ may have been influenced by various investigator or subject biases. ${ }^{33}$ The DATATOP study has generated considerable interest and continuing controversy. ${ }^{34,35}$

Two other small-scale double-blind studies evaluated selegiline monotherapy in a similar manner. ${ }^{36,37}$ Both involved 54 untreated PD subjects randomized to receive placebo or selegiline, $10 \mathrm{mg} /$ day. The study by Tetrud and Langston ${ }^{36}$ found that selegiline slowed the rate of progression in UPDRS ratings by approximately half and without exerting detectable symptomatic anti-parkinsonian effects. With respect to the perceived need for starting dopaminergic therapy (levodopa) in the study participants, the group receiving placebo went for an average of 312 days before reaching that point, compared with 549 days for the subjects receiving selegiline. The study conducted by Myllylä et al. ${ }^{37}$ found that the selegilinetreated subjects also had a significant delay in their determined need for levodopa treatment (median interval: $545 \pm 90$ days), compared with the placebo group (327 \pm 28 days). This study also indicated that parkinsonian disabilities in the selegiline-treated subjects tended to remain milder for up to 12 months; however, the advantage seen with selegiline was lost, in a manner similar to that seen in the DATATOP study.

Selegiline treatment outcomes were also evaluated by the Swedish Parkinson Study Group. ${ }^{38}$ This study randomized 157 de novo PD subjects between treatment arms of selegiline monotherapy $(10 \mathrm{mg} /$ day $)$ or placebo in a double-blind manner. Follow-up at 5 years revealed that selegiline significantly delayed the need for starting dopaminergic treatment. ${ }^{39}$ In contrast to the other selegiline studies mentioned, this trial used an 8-week washout of selegiline, to eliminate possible symptomatic effects of MAO-B inhibition. Clinical outcomes for selegiline monotherapy or combined therapy with levodopa appeared to be improved, compared with treatment with placebo, although such studies present the challenge of sorting out symptomatic effects from neuroprotection. Finally, a more recent randomized double-blind study of placebo or selegiline involving 163 PD subjects indicated that less disease progression developed for subjects who were receiving the MAO-B inhibitor. ${ }^{40}$ Thus, considerable evidence seems to support a disease-modifying effect of selegiline. Nonetheless, the 2004 update of the Movement Disorder Society's task force assessing PD treatments and the American Academy of Neurology subcommittee addressing this matter concluded that available evidence of selegiline helping to prevent clinical progression of PD was insufficient. ${ }^{4-6}$

The MAO-B inhibitory property of selegiline was the initial basis for its consideration in clinical trials of neuroprotection. Unrelated to this effect is evidence in several models of neuronal injury suggesting that the selegiline metabolite desmethylselegiline has additional pharmacological properties similar to those of neurotrophins. ${ }^{41}$

\section{Lazabemide}

The initial results with selegiline prompted consideration of additional MAO-B inhibitors as potential neuroprotective agents. Lazabemide differs from selegiline in several properties: it is a reversible inhibitor of MAO that has greater selectivity for the type B enzyme versus type A and undergoes rapid clearance after discontinuation. Furthermore, lazabemide is not a propargylamine compound and is not metabolized to amphetamine. ${ }^{42,43}$ The various studies of selegiline as a disease-modifying therapy had to contend with the possibility of several potentially confounding factors, especially the possibility that symptomatic effects gave the appearance of arrest in disease progression. Even though D-amphetamine metabolites of selegiline were regarded as unlikely to confer an anti-parkinsonian effect, this possibility remained unanswered. Because lazabemide could be washed out in a short time, this drug provided an opportunity to evaluate the effect of MAO-B inhibition versus other pharmacological properties that seemed to confound the selegiline studies.

Initial studies with lazabemide in otherwise untreated PD subjects revealed that its symptomatic effects were similar to those of selegiline. ${ }^{42,43} \mathrm{Next}$, a trial assessing its possible neuroprotective actions was conducted in a randomized, double-blind study lasting up to 1 year. ${ }^{44}$ This placebo-controlled investigation enrolled $321 \mathrm{PD}$ 
subjects who were not receiving symptomatic therapy and who received either placebo or lazabemide (dosage range, $25-200 \mathrm{mg} /$ day). The smaller number of subjects enrolled, compared with the DATATOP trial, was based on considerations of study power needed to achieve clinically meaningful results against the natural history of PD progression. In this study, the primary endpoint was the same as the DATATOP trial (the determination of functional disability necessitating the start of symptomatic therapy with levodopa or an equivalent therapy). After 12 months of lazabemide treatment, the outcome was closely similar to the findings of DATATOP. Although lazabemide provided only a small improvement in total UPDRS score at 6 months into the study $(\sim 2$ units), the repeated assessments of need-for-levodopa endpoint were subjected to a Kaplan-Meier analysis and it was found that the drug reduced this outcome by $51 \%$ $(p=0.008){ }^{44}$

The implications of the Parkinson Study Group trial, ${ }^{44}$ taken together with the results of the various selegiline trials, were that MAO-B inhibition (and not the additional pharmacological properties of selegiline) was responsible for the delay in reaching the need for the start of dopaminergic treatment and for relative improvements in the overall worsening of UPDRS scores. The lazabemide study provided a better opportunity for conducting a valid comparison of baseline to post-treatment PD status after drug washout and lacking the possible carryover of anti-parkinsonian effect (which continued with selegiline even several weeks after washout). ${ }^{10}$ Despite the positive findings of this study, the study sponsor chose not to continue development of lazabemide for PD.

\section{Rasagiline}

Rasagiline is a highly selective MAO-B inhibitor. It shares, with selegiline, a propargylamine structure and irreversible inhibition. ${ }^{45}$ Rasagiline enhances release of dopamine, in addition to retarding its catabolism, and antagonizes three cellular processes that are involved in the cascade of events leading to apoptosis: intranuclear translocation of the glycolytic enzyme glyceraldehyde3-phosphate dehydrogenase, induction of bcl-2, and activation of mitochondrial permeability transition. ${ }^{46,47}$ Another effect seen in experimental models of parkinsonian degeneration is averting apoptosis by means of inducing GDNF. Rasagiline also blocks actions of an endogenous neurotoxin that is a condensation product of oxidized dopamine, $N$-methyl- $(R)$-salsolinol. Animal studies have shown functional recovery is elicited by rasagiline even when administered after exposure to the neurotoxin 1-methyl-4-phenyl-1,2,3,6-tetrahydropyridine (MPTP). ${ }^{48}$

The TEMPO trial ${ }^{49}$ (Rasagiline Mesylate [TVP-1012] in Early Monotherapy for Parkinson's Disease Outpatients) was conducted in 404 early, untreated PD patients for 26 weeks as a randomized, double-blind, placebo- controlled clinical trial using rasagiline at doses of 1 and $2 \mathrm{mg} /$ day. The primary efficacy measure was the net change in total UPDRS score from baseline to 6 months; a secondary endpoint, similar to that of the DATATOP study, was the need for starting levodopa because of advancing disability. A novel feature of this study was its delayed-start design, used in an effort to differentiate the symptomatic effects of MAO-B inhibition from its possible neuroprotective actions. This differentiation was determined in the second 26-week phase of the study, in which the group initially receiving placebo was switched to rasagiline, $2 \mathrm{mg} /$ day.

In the first phase of the study, both rasagiline doses produced similar symptomatic benefits-improvement in UPDRS scores. The placebo group did not lessen their score. The separation between the rasagiline and placebo-treated groups continued through the 26-week study $(p=0.02)$. Once receiving rasagiline, after 6 months, the group initially receiving placebo experienced a similar degree of symptomatic effect as did the group initially receiving the drug; however, the delayed-start group never caught up to the level of UPDRS improvement in the group initially receiving rasagiline. This latter observation suggests more than a symptomatic effect. After 12 months of continued rasagiline treatment, the group initially receiving the active drug continued to maintain the same degree of improvement over the delayed-start group.

The results of the TEMPO trial were thus in favor of a disease-modifying action. Over the first 6 months of the study, both doses of rasagiline yielded similar improvements, approximately 4 UPDRS units $(p<0.001)$. In the DATATOP trial, selegiline-treated subjects also achieved an effect of approximately this magnitude at 6 months. ${ }^{9,10}$ Unlike DATATOP, however, a KaplanMeier survival analysis of TEMPO did not find a statistically significant difference between placebo and either rasagiline dose for the secondary endpoint (delay in the need for starting levodopa or a comparable treatment for emerging parkinsonian disability). During the 6-month trial, 23 of 138 subjects in the placebo group (16.7\%) reached the secondary endpoint of need for levodopa therapy, compared with 15 rasagiline-treated subjects $(11.2 \%)$ receiving $1 \mathrm{mg} /$ day and 22 receiving $2 \mathrm{mg} /$ day $(16.7 \%)$. The TEMPO study also reported that both doses of rasagiline gave a statistically significant improvement in PDQUALIF ratings (a quality of life measure), and in the UPDRS Parts 2 and 3 (Activities of Daily Living and Motor subscales). ${ }^{49}$

As a follow-up to the TEMPO study, a larger scale and longer randomized, placebo-controlled investigation of rasagiline monotherapy ( 1 or $2 \mathrm{mg} / \mathrm{day}$ ) in approximately 1100 subjects with mild PD is underway. This study, termed the ADAGIO trial (Attenuation of Disease Progression with Rasagiline Once-Daily), will also apply a 
delayed-start design in an effort to differentiate the possible disease-modifying effects conferred by 36 weeks of initial rasagiline treatment. The second phase of the study, in which subjects initially receiving placebo will be randomized to rasagiline at 1 or $2 \mathrm{mg} / \mathrm{day}$, will also last 36 weeks. Results are planned to be reported later in 2008.

The effectiveness of selegiline, lazabemide, and rasagiline as disease-modifying agents provides a focus on their shared property of MAO-B inhibition. Additional potentially protective pharmacological properties of propargylamine compounds that are unrelated to MAO-B inhibition, however, have also been shown in laboratory models of neurodegeneration through apoptosis. ${ }^{47,50}$ Whether another MAO-B inhibitor currently under development, safinamide, ${ }^{51}$ might also possess disease-modifying effects remains to be studied. A compound derived from the structure of selegiline, TCH346, was studied as a possible neuroprotectant in PD but yielded negative results ${ }^{52}$ (discussed under Antiapoptotic Compounds).

\section{DOPAMINERGIC DRUGS}

Although developed for their symptomatic actions in $\mathrm{PD}$, several drugs with dopaminergic properties have also provided evidence for a neuroprotective effect. In support of disease-modifying effects are randomized clinical studies conducted with two dopaminergic agonists, pramipexole (CALM-PD) $)^{53-55}$ and ropinirole (REAL-PET) ${ }^{56}$ and a study investigating levodopa (ELLDOPA) ${ }^{16}$ In a study planned to evaluate possible disease-modifying effects from 3 years of pergolide versus levodopa treatments (PELMOPET: Pergolide versus L-dopa as Monotherapy and Positron Emission Tomography), ${ }^{57}$ the disease progression analysis could not be performed because of noncompliance on the part of investigators and research subjects; subsequently pergolide was withdrawn from the market. Another randomized controlled clinical trial (CABASER 09 study), investigating possible disease modification by cabergoline, has been reported to have shown benefits of this dopaminergic agonist, compared with levodopa therapy, although its results have not appeared as a peer-reviewed publication.

The first clues suggestive of beneficial effects of dopaminergic agonists on neurons came from a study in rats, in which daily oral intake of pergolide lessened age-related attrition of SN neurons. ${ }^{58}$ Studies with pramipexole have demonstrated a number of potentially protective actions against oxidative stress and the influence on dopaminergic neurons of various experimental toxins, including methamphetamine, 3-acetylpyridine, 6-hydroxydopamine (6-OHDA), and MPTP. ${ }^{59-72}$ The mechanisms contributing to the protective actions of pramipexole have not been defined, although activation of the D3 dopamine receptor was suggested from one study ${ }^{69}$ and blocking the cascade of apoptosis in others. These latter effects appeared to be derived from enhanced expression of bcl-2 protein in neuronal dendritic processes or to other actions unrelated to the dopaminergic actions of the drug. ${ }^{59,60,71,72}$ Furthermore, studies investigating a stereoisomer of pramipexole that is inactive at dopamine receptors have shown it exerts neuroprotective properties. ${ }^{64}$ In mice, the dopaminergic agonist ropinirole also enhances mechanisms against oxidative stress and exerts a protective action against 6-OHDA-induced loss of nigrostriatal dopaminergic projections. ${ }^{73}$ Taken together, the implications of these studies are that dopaminergic agonists, either on the basis of dopaminergic stimulation or other properties, can act to mediate recovery of dopaminergic nigrostriatal neurons from the effects of various toxic mechanisms.

The neuroprotective implications of the laboratory research with dopaminergic compounds have inspired several clinical trials, and the question of the effects of levodopa on disease progression prompted formal analysis by means of a controlled clinical study. The challenge in conducting such studies of disease modification is to separate the confounding symptomatic effects. One approach has been to rely on a surrogate biomarker specific for degeneration in dopaminergic nigrostriatal projections. For this purpose, two major techniques have included the use of neuroimaging: positron emission tomography (PET) with $\left[{ }^{18} \mathrm{~F}\right]$ fluorodopa, and SPECT with the labeled dopamine transporter ligand $\left[{ }^{123} \mathrm{I}\right] 2 \beta$-carboxymethoxy-3 $\beta$ (4-iodophenyl)tropane) ([ $\left.\left.{ }^{123} \mathrm{I}\right]-\beta-\mathrm{CIT}\right) .{ }^{18,21-23,74}$

With use of these techniques, disease progression can apparently be inferred even when PD patients continue to receive symptomatic therapy. In each instance, the assumption is that caudate and putamen images provide a direct correlate to the number of dopaminergic projections. There are several caveats for using neuroimaging markers as surrogates for gauging disease progression. Foremost among these is the need to exclude the possibility that dopaminergic treatments might alter the $\left[{ }^{18}\right.$ F]fluorodopa uptake (with PET) or dopamine transporter binding (with SPECT) in a manner that would prevent a valid comparison with a subject's baseline status. $^{22}$

\section{Pramipexole}

The CALM-PD trial (Comparison of the Agonist Pramipexole with Levodopa on Motor Complications of Parkinson's Disease) randomized 301 mildly affected PD subjects between regimens starting with either levodopa or pramipexole. ${ }^{53-55}$ If needed, supplemental levodopa could also be used. CALM-PD participants underwent evaluations of antiparkinsonian efficacy and development of motor fluctuations for up to 4.5 years. A 
group of 82 subjects underwent SPECT neuroimaging using the labeled dopamine transporter ligand $\left[{ }^{123} \mathrm{I}\right]-\beta$ CIT, planned for three post-treatment time points up to 46 months after baseline assessments. ${ }^{55}$ The results of SPECT studies demonstrated less decline in striatal dopamine transporter binding for the group initially randomized to pramipexole, compared with subjects assigned to levodopa treatment. In comparison with baseline scans, $\left[{ }^{123} \mathrm{I}\right]-\beta$-CIT SPECT imaging revealed reductions in dopamine transporter binding of $7.1 \pm$ $9.0 \%$ versus $13.5 \pm 9.6 \%$ at 22 months, $10.9 \pm 11.8 \%$ versus $19.6 \pm 12.4 \%$ at 34 months, and $16.0 \pm 13.3 \%$ versus $25.5 \pm 14.1 \%$ at 46 months (means $\pm \mathrm{SD}$; all $p$-values $\leq 0.01$ ). The diminished rate of decline in the pramipexole-treated group suggested a protective action (although, alternatively, levodopa treatment might have accelerated disease progression).

The lack of an untreated control group has hampered interpretation of CALM-PD results, as have lingering concerns that the dopaminergic treatments might have pharmacologically altered binding of $\left[{ }^{123} \mathrm{I}\right]-\beta$-CIT. ${ }^{21-23}$ Clinical ratings conducted after a pramipexole or levodopa washout of at least 12 hours permitted measurements of UPDRS scores in an unmedicated state. These showed no differences between the two groups; however, the short duration of washout for these symptomatic treatments is likely to have been inadequate for valid comparisons to baseline. Many of the subjects originally assigned to pramipexole monotherapy eventually added levodopa to their treatment regimen. The results of the pramipexole-only treatment subgroup have not been reported. Furthermore, some of the subjects had initial $\left[{ }^{123} \mathrm{I}\right]-\beta$-CIT binding density that was not typical of a diagnosis of PD, but these subjects were not excluded from the analysis.

\section{Ropinirole}

Two investigations were conducted to compare outcomes of ropinirole and levodopa treatment in PD subjects. The first involved $45 \mathrm{PD}$ subjects randomized to either levodopa (up to $1200 \mathrm{mg} /$ day) or ropinirole (up to $24 \mathrm{mg} /$ day). ${ }^{75}\left[{ }^{18} \mathrm{~F}\right]$ fluorodopa PET studies of tracer uptake by the putamen were conducted for comparison with baseline scans. After 24 months, the reduction in uptake was $13 \%$ for the 28 ropinirole-treated subjects, compared with an $18 \%$ reduction for the 9 receiving levodopa. These changes were not statistically significant, and the study was underpowered for detecting a difference.

The REAL-PET trial (ReQuip [ropinirole] as Early Therapy versus L-Dopa-PET) randomized 86 mildly affected PD subjects to initial monotherapy regimens of either levodopa or ropinirole. ${ }^{56}$ Unlike the CALM-PD study, the REAL-PET study used the baseline results to validate the diagnosis of PD. If subjects lacked evidence for $\left[{ }^{18} \mathrm{~F}\right]$ fluorodopa PET neuroimaging changes typical of PD, their imaging data were excluded from analysis. The ropinirole-treated group showed evidence for less disease progression than the levodopa group. Over the 2-year study interval, the ropinirole group's change from baseline in $\left[{ }^{18} \mathrm{~F}\right]$-fluorodopa uptake in the putamen was a $13 \%$ loss in label uptake, compared with a $20 \%$ reduction in the group randomized to levodopa $(p=0.022)$.

With statistical parametric mapping, two brain regions of interest were used to detect significantly slower disease progression with ropinirole. Imaging of the putamen showed a $14 \%$ reduction in disease progression with ropinirole treatment, compared with a reduction by $20 \%$ for treatment with levodopa alone $(p=0.034)$. Elsewhere in the brain, imaging in the substantia nigra for the ropinirole group revealed a $+3 \%$ change with ropinirole, compared with reduction by $-8 \%$ with levodopa $(p=$ 0.035). Overall, these results support a disease-modifying effect of ropinirole. As for the CALM-PD study, an alternative conclusion could be that levodopa treatment had a disease-accelerating effect.

If both ropinirole and pramipexole did exert a neuroprotective action, the similar study results support the possibility of a "class effect" conferred by dopaminergic agonists but not levodopa. Several reviews of these data have cited the need for further validation of the technique and an untreated control group before meaningful conclusions can be drawn. The effects of levodopa were especially intriguing, and set the stage for study of its influence on progression of PD.

\section{Levodopa}

Long-standing concerns that levodopa might be neurotoxic have been difficult to evaluate because of the pervasive use of this drug as a chronic symptomatic therapy. Levodopa generates oxyradicals and has other possible mechanisms for causing neuronal degeneration, suggested by in vitro experiments showing the generation of an excitotoxin, trihydroxyphenylalanine, ${ }^{76}$ and the neurotoxin 6-OHDA. ${ }^{77}$ Nonetheless, evidence is lacking for levodopa toxicity in the treatment of PD patients. ${ }^{78}$ Because levodopa has antioxidant effects in the striatum, ${ }^{79}$ there has also been a rationale to explore a beneficial effect.

The possibility that levodopa might have a diseasemodifying effect was investigated in the ELLDOPA trial (Earlier versus Later Levodopa), conducted over 40 weeks. ${ }^{16}$ Three daily intake regimens $(150,300$, and 600 $\mathrm{mg} / \mathrm{day}$ ) of levodopa or placebo were administered to the 361 randomized, mildly affected PD subjects. After 40 weeks, subjects underwent a 2-week washout of study medication for comparison with baseline status. Clinical ratings supported an apparent protective effect of levodopa, compared with placebo. UPDRS total scores revealed a mean difference between baseline and end- 
of-study ratings of 7.8 units for the placebo group of 1.9 units at $150 \mathrm{mg} / \mathrm{day}, 1.9$ units at $300 \mathrm{mg} /$ day), and -1.4 units at $600 \mathrm{mg}$ /day (all comparisons, $p<$ $0.001)$. These findings were compatible with a doserelated beneficial disease modification. However, interpretation of the data required that all symptomatic actions of levodopa were effectively washed out. This assumption, though reasonable, was questioned by analysis of data from the levodopa-treatment extension of the DATATOP study, which concluded that a 2-week washout of levodopa may be inadequate to eliminate all anti-parkinsonian effects. ${ }^{80}$ The latter study concluded that a neuroprotective effect of levodopa treatment seemed likely.

The results of this study were further confounded by neuroimaging results. An ELLDOPA subset of 142 subjects underwent $\left[{ }^{123} \mathrm{I}\right]-\beta$-CIT SPECT neuroimaging, to assess change in striatal dopamine transporter density between baseline and week 40. Although the clinical results suggested dose-related reduction in disease progression, the $\left[{ }^{123} \mathrm{I}\right]-\beta$-CIT results suggested just the opposite. The data revealed the mean percent-decline was significantly greater during levodopa treatments than with placebo, which declined by $1.4 \%$ during the trial. Reductions among the various levodopa doses were $6 \%$ (150 mg/day), $4 \%$ (300 mg/day), and $7.2 \%$ (600 mg/ day). Excluded from the analysis were 19 subjects with no dopaminergic deficits on the baseline SPECT scans (and, hence, only clinical evidence for the diagnosis of $\mathrm{PD})$. The reason for their exclusion was the presumption that the mild parkinsonian features they exhibited did not represent true $\mathrm{PD}$, despite the clinical impression of an experienced PD specialist who enrolled these subjects. A post hoc analysis of the ELLDOPA data including only subjects with baseline SPECT data compatible with PD found that the $600 \mathrm{mg} /$ day levodopa regimen yielded the greatest reduction in $\left[{ }^{123} \mathrm{I}\right]-\beta$-CIT binding.

Parkinsonian subjects with normal dopamine transporter site density (constituting 13\% of the total group studied by $\left[{ }^{123} \mathrm{I}\right]-\beta$-CIT SPECT) provide an important insight into neuroprotective trials that use only clinical criteria for the choice of appropriate study candidates. The apparent dose-related worsening of PD (as judged by the increased severity of the nigrostriatal lesion on $\left[{ }^{123} \mathrm{I}\right]-\beta$-CIT neuroimaging) suggested an effect opposite to the clinical observations. Concerns that chronic levodopa use might modify the number of sites or binding properties at the dopamine transporter was not supported by an investigation of $\left[{ }^{123} \mathrm{I}\right]-\beta$-CIT SPECT imaging after levodopa washout. ${ }^{23}$ However, the simple question as to whether levodopa has an influence at slowing or accelerating the progression of PD will require further analysis beyond the ELLDOPA study.

\section{ANTIOXIDANT STRATEGIES}

The theme of the DATATOP trial was protection against the generation or actions of oxyradicals. ${ }^{8-10} \mathrm{Al}-$ though several compounds with antioxidant properties have been considered for clinical investigation, only $\alpha$-tocopherol has undergone testing. Coenzyme $\mathrm{Q}_{10}$ and creatine (discussed in the next section) also may confer antioxidant actions, in addition to their other intended effects on mitochondrial complex I metabolism. $\alpha$-Tocopherol, a chain-breaking antioxidant that enters into lipid-soluble cellular regions such as biological membranes, acts by quenching oxyradical species. There is no evidence for deficiency of $\alpha$-tocopherol in PD, and severe deficiency states do not lead to parkinsonism. Nevertheless, this naturally occurring antioxidant offered a safe and promising option for testing the hypothesis of oxidative stress in the pathogenesis of PD.

The notion of using antioxidant vitamins (vitamin $\mathrm{E}$ $3200 \mathrm{IU} /$ day and vitamin C $3000 \mathrm{mg} /$ day) as potential neuroprotectants was tested initially in an open-label study. No conclusions as to effectiveness were drawn. ${ }^{81}$ In the DATATOP study, 800 enrolled subjects were randomized to regimens of placebo, 2000 IU of $\alpha$-tocopherol daily, selegiline, and both $\alpha$-tocopherol and selegiline. in a $2 \times 2$ factorial design..$^{8-10}$ The 400 subjects receiving $\alpha$-tocopherol or matching placebo were followed for 2 years without breaking of the study "blind" (even though the selegiline-placebo portion of the DATATOP study was prematurely ended, and most subjects switched to open-label selegiline). On the basis of the same study endpoints used to assess the effect of selegiline, there was no evidence for a disease-modifying effect. $^{10}$

\section{MITOCHONDRIAL ENERGY ENHANCEMENT}

One of the few systemic markers discovered for PD is that of altered mitochondrial function. Mitochondria of SN, platelets, and skeletal muscle in PD possess reduced activity of the first step of the mitochondrial electron transport chain, complex I. ${ }^{11,12}$ This is also found in parkinsonism associated with mutations of the PINKI and PARK7 (previously $D J-1$ ) genes. ${ }^{82}$ An animal model of parkinsonism can be created by means of rotenone, a toxin that which selectively inhibits the electron chain functions of mitochondrial metabolism at complex I. It is not known whether this abnormality contributes to pathogenesis, or is merely an epiphenomenon (also found in several other neurodegenerative disorders) ${ }^{12}$; however, this well-validated finding prompted therapeutic interventions that might enhance the activity of mitochondrial complex I. Coenzyme $\mathrm{Q}_{10}$ (also known as ubiquinone) is an essential cofactor serving as an electron acceptor for mitochondrial complex I. Coenzyme 
Q10 is also a potent antioxidant in lipid membranes and mitochondria. Several studies have provided evidence for its potential in averting progression of PD. ${ }^{83,84}$

Another pharmacologic strategy for targeting the defect in mitochondrial complex I is the augmentation of brain creatine concentration. Creatine serves as a precursor for conversion to the energy intermediate phosphocreatine, which in mitochondria transfers phosphoryl groups for ATP synthesis. The effect of increasing creatine intake is an enhancement of phosphocreatine formation. Ultimately, the result is reduction in oxidative stress through stabilization of mitochondrial creatine kinase. Creatine kinase is involved in inhibiting the opening of the mitochondrial transition pore (a mechanism involved in initiating apoptosis). Hence, the potential improvement of mitochondrial metabolism and downregulation of a putative neurodegenerative mechanism could be the result of supplementation with creatine. Only limited preclinical evidence has guided decisions to go ahead with creatine clinical trials in PD. ${ }^{11,18}$ For mice treated with MPTP, supplementing their diet with creatine for 2 weeks before administration of the neurotoxin led to less damage to dopaminergic SN neurons. ${ }^{85}$

\section{Coenzyme $\mathbf{Q}_{10}$}

A clinical trial was completed for coenzyme $\mathrm{Q}_{10}$, using dosages of 300,600 , and $1200 \mathrm{mg} /$ day. This placebocontrolled, randomized investigation enrolled 80 otherwise untreated PD subjects for 16 months of study drug administration. The primary endpoint was the perceived need for the start of levodopa, and a secondary endpoint was change in UPDRS score. A positive outcome was found for subjects receiving $1200 \mathrm{mg}$ of coenzyme $\mathrm{Q}_{10}$ per day (but not the 300 and $600 \mathrm{mg} /$ day regimens). This consisted of an improvement of 6.7 points $(p=0.0416)$ in the adjusted mean score for total UPDRS score. The comparison with placebo treatment was not statistically significant, although a prespecified endpoint of a positive trend was achieved. ${ }^{18}$ Review of the data revealed that most of the benefit observed with the highest dose of coenzyme $\mathrm{Q}_{10}$ was derived from its effect on the score for UPDRS Part 2-Activities of Daily Living, rather than to improvement in the motor examination for parkinsonism, UPDRS Part 3-Motor Score.

These intriguing (but underpowered) results provided great interest in linking the mitochondrial electron chain transfer defect with a possibly correctable mechanism for causation of PD. Because coenzyme $\mathrm{Q}_{10}$ also has antioxidant properties, other actions accounting for its disease-modifying effects are plausible. The authors reporting these findings emphasized that further study of the effects of coenzyme $\mathrm{Q}_{10}$ were warranted before any recommendation as to therapeutic use could be made. ${ }^{18}$ The high dosage of coenzyme $\mathrm{Q}_{10}$ needed for clinical trials is a logistical problem. Improved bioavailability with new preparations ${ }^{86}$ will help to make subsequent studies more feasible.

Further investigation of coenzyme $\mathrm{Q}_{10}$ was performed in a NET-PD clinical trial using $2400 \mathrm{mg} /$ day. ${ }^{81}$ That dose was one arm of a randomized, placebo-controlled futility trial linked with investigation of GPI-1485. Subjects who did not need and were not receiving symptomatic treatment of PD were randomized 1:1:1 among the three treatment assignments. The primary outcome measure was the change in total UPDRS score over 12 months from baseline to disability requiring dopaminergic therapy (or not). A number of ratings were conducted in the testing battery in addition to the UPDRS, including measures of depression, cognitive function, quality of life, and disability. Five time points after baseline (1, 3, 6,9 , and 12 months) were used for the testing battery assessing treatment effects. A total of 213 subjects were randomized to the three treatment arms. The mean change in UPDRS score in the group that received coenzyme $\mathrm{Q}_{10}$ was 7.52 (SD: 8.87). Compared with the preset threshold value of 7.46 for progression of $\mathrm{PD}$, found in a previous placebo-controlled study of otherwise untreated parkinsonism (DATATOP ${ }^{7,8}$ ), coenzyme $\mathrm{Q}_{10}$ treatment could not be rejected as futile $(p \leq 0.1)$. Hence, the study recommended that an additional definitive evaluation was warranted. ${ }^{83}$ In support of this conclusion (but independent of the NINDS NET-PD consortium), a randomized placebo-controlled clinical trial with coenzyme $\mathrm{Q}_{10}$ in mild $\mathrm{PD}$ is currently underway in North America, using a dose of $2400 \mathrm{mg} /$ day.

Futility trials offer conclusions only as good as the guidance offered by realistic expectations of meaningful clinical effect. With respect to the coenzyme $\mathrm{Q}_{10}$ and GPI-1485 study, ${ }^{27,83}$ the results from the placebo treatment arm in DATATOP were used to create the threshold for assessment of possible futility. However, exploratory studies of the data from the coenzyme $\mathrm{Q}_{10}$ and GPI-1485 experience raised the possibility that investigators may have applied different standards for determination of endpoint, compared with investigators in the DATATOP study (which was conducted almost two decades previously). In addition, if disease progression results from the PRECEPT trial of CEP- $1347^{87}$ (discussed in its own section) had instead been used as the comparator for the effects of coenzyme $\mathrm{Q}_{10}$, then this exploratory analysis would have suggested that a trial of coenzyme $\mathrm{Q}_{10}$ as a neuroprotectant would be futile.

\section{Creatine}

A multicenter, randomized, placebo-controlled clinical trial in otherwise untreated PD subjects was conducted using creatine. This NET-PD investigation involved a futility study design, conducted with minocycline as another experimental treatment, to learn if a larger study would be futile for disease modification. ${ }^{88}$ The primary 
outcome determinant was change in total UPDRS from baseline to the first occurrence of either 1) the time when sufficient disability had evolved requiring the start of symptomatic therapy, or 2) 12 months without the need for such treatment. The prespecified threshold for determining therapeutic effect was derived from rate of decline in the DATATOP study in total UPDRS score over time for subjects not receiving selegiline. A 30\% reduction was chosen as the signal for a clinically meaningful neuroprotective effect to counter a conclusion of futility at $p \leq 0.1$.

Mildly affected and unmedicated PD subjects $(n=$ 200) were randomized 1:1:1 among placebo and the two treatment arms, creatine ( $10 \mathrm{~g} /$ day $)$ and minocycline (200 $\mathrm{mg} /$ day). Neither intervention reached prespecified futility criteria. Although side effects occurred with each, and minocycline was not as well-tolerated as creatine, neither treatment produced serious adverse outcomes. A methodological issue (which also arose for the coenzyme $\mathrm{Q}_{10}$ and GPI-1485 study ${ }^{83}$ ) had to do with the placebo-treatment group chosen as a historical control for the purpose of gauging the futility threshold endpoint from the DATATOP study, as already discussed regarding coenzyme $\mathrm{Q}_{10}{ }^{88}$

Another placebo-controlled study evaluating creatine supplementation was conducted in 60 subjects. ${ }^{89}$ This study differed from the NET-PD trial in that subjects were receiving levodopa and other anti-parkinsonian therapy (except for selegiline). The assessment of change over the 2-year study was made by comparing clinical ratings while subjects were receiving the same dosages of PD medications at the end of the study as were taken initially. The results of this trial revealed that the creatine supplementation regimen (loading dose of $20 \mathrm{mg} / \mathrm{day}, 2$ $\mathrm{g} /$ day for 6 months, and then $4 \mathrm{~g} /$ day until the end of the study) was associated with slight benefit in the UPDRS Part 1-Mental Status score. This improvement occurred primarily with the single item relating to depression. Although no improvements were detected in UPDRS Part 3-Motor Score scores, the creatine-treated subjects tended to require less increase of dopaminergic therapy dose over time. However, due to a number of methodological issues including confounding effects of symptomatic therapy and the lack of standardization in dose adjustment, the significance of these results is uncertain. This study also used SPECT neuroimaging with a dopamine transporter ligand, which revealed no treatment effect of creatine between baseline and the end of the study.

\section{ANTIAPOPTOTIC COMPOUNDS}

Several lines of evidence have pointed to the activation of apoptosis as a possible mechanism for neurodegeneration in PD. On this basis, the search for antiapop- totic interventions led to proposals for study of three compounds differing in how they interact with pro-apoptotic mechanisms. Although there has been support for this treatment strategy in some animal models of toxininduced parkinsonism, there is no consensus in the research community that apoptosis is a primary mechanism of neurodegeneration, or even a common final pathway. ${ }^{90}$

\section{Minocycline}

Minocycline has been extensively studied because of its promise in treating neurodegenerative disease. In rodent models of parkinsonism induced by 6-OHDA and MPTP, pretreatment with minocycline improved survival of dopaminergic SN neurons. ${ }^{91}$ Minocycline inhibits the activation of microglia, which is a prominent feature in the brain of PD patients and in experimental neurotoxin models. ${ }^{92,93}$ This drug also acts to lessen factors that mediate apoptosis, such as caspase-I. ${ }^{92}$ Although these properties seem to be in favor of minocycline providing a possible neuroprotective effect in PD, preclinical results have not been consistent in support of this possibility. ${ }^{94}$

As one of the treatment arms in the NET-PD randomized, double-blind clinical trial also investigating creatine, minocycline $100 \mathrm{mg}$ twice daily was also evaluated as a disease-modifying intervention subjected to a futility analysis. ${ }^{88}$ The primary outcome measure was change in total UPDRS score from baseline to either a determination of disability requiring dopaminergic therapy or 1 year, whichever was first. The mean change for the minocycline group was $7.09(\mathrm{SD}=8.71)$, compared with the threshold value of 7.46 (70\% of the historical DATATOP study rate of UPDRS progression ${ }^{9,10}$ ). As a result, the prespecified hypothesis of futility for further study could not be rejected. ${ }^{88}$ Although minocycline is somewhat problematic as a treatment because of side effects and tolerability, the results of this investigation warrant considerations of additional study (with consideration for the caveats expressed in recent preclinical research). ${ }^{94}$

\section{TCH346}

Another candidate in the search for antiapoptotic interventions was TCH346 (also designated CGP 3466 or CGP 3466B in various publications). TCH346 is a novel compound developed because of its shared structural similarities with selegiline. TCH346 does not inhibit MAO-B, however, and, unlike selegiline, is not metabolized to amphetamine compounds. It acts to inhibit a key step in age-induced neuronal apoptosis through binding to the glycolytic enzyme glyceraldehyde-3-phosphate dehydrogenase (which is a binding site also for selegiline and rasagiline). ${ }^{95,96}$ Cell culture studies with PC12 and human neuroblastoma cell lines have shown increased survival with this agent. ${ }^{97}$ In rhesus monkeys 
exposed to MPTP, near-complete protection against the development of motor impairment was achieved when TCH346 administration was started 2 hours after the second MPTP infusion and continued for 2 weeks. ${ }^{98}$ Histological analysis revealed that TCH346 treatment led to sparing of the usual loss caused by MPTP in dopaminergic SN neurons and their projections to the striatum. Together with the findings from cell culture experiments, these results were the impetus for a large multicenter clinical trial assessing neuroprotection in PD.

For the 301 early, untreated PD subjects enrolled, randomized treatment assignments were made to placebo or one of three oral doses of TCH346 $(0.5,2.5$, or 10 $\mathrm{mg} /$ day) and continued over 12 to 18 months. ${ }^{52}$ After the treatment period, each subject had a 4-week washout. The primary study endpoint was time to requirement of dopaminergic therapy because of emerging disability. Other outcome measures included the rate of decline in total UPDRS scores and change in a quality of life evaluation (PDQ-39). Most subjects completed the trial, and no toxicity was encountered. Over the course of the study, approximately one-third of each treatment arm met primary endpoint criteria, including placebo. The rate of annual change in UPDRS scores or PDQ-39 did not reveal any evidence for a neuroprotective effect. ${ }^{52}$

\section{CEP-1347}

CEP-1347 (also designated KT7515) is a semisynthetic derivative of a bacterial fermentation product termed K252, which acts as an inhibitor of mixed lineage kinase-3. This enzyme is a major component in the transcription factor c-Jun-mediated terminal kinase signaling pathway, which is involved in apoptotic death of neurons. The cascade of events through this pathway has been hypothesized to be a mechanism by which SN neurons are lost in PD. Several laboratory studies involving neuronal PC12 cells, sympathetic neurons, and other in vivo models of neurodegeneration ${ }^{99}$ supported this potential. Mice and monkeys exposed to MPTP have enhanced survival of SN neurons with this compound. ${ }^{99}$ In 30 untreated PD subjects participating in an initial feasibility study, CEP-1347 was safe, well tolerated, and demonstrated no symptomatic effects in a randomized, placebo-controlled study using $50 \mathrm{mg}$ twice daily. ${ }^{100}$

A larger scale investigation followed. The PRECEPT study ${ }^{87}$ recruited untreated PD subjects lacking current or imminent need for anti-parkinsonian treatment. The primary endpoint was again the need for the start of dopaminergic treatment. Secondary endpoints included UPDRS changes and $\left[{ }^{123} \mathrm{I}\right]-\beta$-CIT SPECT scans.

After implementation of this placebo-controlled trial, which enrolled 806 subjects with three daily dose regimens of CEP-1347, the study was halted as a result of an interim futility analysis. At this point, $57 \%$ of subjects receiving placebo had reached the primary endpoint, compared with $65 \%$ of subjects receiving CEP-1347 20 $\mathrm{mg} / \mathrm{day}, 59 \%$ of subjects receiving $50 \mathrm{mg} / \mathrm{day}$, and $64 \%$ of subjects receiving $100 \mathrm{mg} /$ day. ${ }^{87}$ These results were comparable to the lack of effect also found in determinations of total UPDRS score and in changes between baseline and final $\left[{ }^{123} \mathrm{I}\right]-\beta$-CIT SPECT scans. Most of the subjects who underwent neuroimaging at the start of the study showed marked reductions in dopamine transporter binding in the putamen that were compatible with PDthat is, were less than $80 \%$ of the lowest age-expected binding by $\left[{ }^{123} \mathrm{I}\right]-\beta$-CIT. However, 91 of the 799 subjects who were scanned $(11.4 \%)$ did not meet these criteria. With the consideration that these subjects might have confounded the study findings because of a questionable diagnosis of PD, a post hoc analysis was conducted and revealed no change in the pattern or numeric trends of the CEP-1347 treatment effects that would change conclusions made with the entire subject group.

\section{ANTIGLUTAMATERGIC AGENTS}

Because glutamate can act as an excitotoxin contributing to neuronal damage, ${ }^{101}$ one rationale for PD neuroprotection has been to block glutamate neurotransmission in the SN. This amino acid neuromodulator has been under consideration as a contributing factor for a number of neurodegenerative disorders. Studies using animal models of parkinsonism have suggested that glutamate can confer excitotoxic damage via NMDA receptors. ${ }^{102}$

\section{Riluzole}

This compound has been shown to demonstrate limited but definite effectiveness in slowing deterioration of amyotrophic lateral sclerosis and has been FDA-approved for this use. Riluzole acts by blocking the presynaptic release of glutamate. Unlike other compounds that are potent glutamate blockers and that can cause significant CNS toxicity, riluzole is well tolerated.

A large multicenter randomized clinical trial with riluzole $(100 \mathrm{mg} /$ day $)$ and placebo was conducted to determine if a possible neuroprotective effect exists. The study was structured with endpoints similar to those previously used in clinical trials with selegiline: the need to start dopaminergic therapy, and UPDRS scores. After an interim analysis revealed no evidence for a neuroprotective effect, this trial was halted, and there has been only limited reporting of results. ${ }^{103}$ Another investigation of this compound was undertaken in 20 subjects, followed over 6 months as an exploratory study. The study used a variety of assessments, some of which pointed to a trend for slowing of disease progression, but the results were essentially inconclusive because the study was underpowered and of short duration. ${ }^{104}$ 


\section{CONCLUSIONS}

Over the past two decades, neuroprotection has been the goal of several randomized, placebo-controlled clinical trials, some involving hundreds of subjects and lasting more than 12 months. A few have provided intriguing evidence for disease modification through clinical ratings or surrogate markers. However, even those treatments with scientifically compelling results showed limited benefits, leading to questioning of their practical application. For example, the modest improvement offered by $1200 \mathrm{mg} /$ day of coenzyme $\mathrm{Q}_{10}$ on UPDRS scores for activities of living was not, in the opinion of the study authors, clinically meaningful enough to merit recommending this compound in standard practice as a neuroprotective treatment. ${ }^{18}$ Testing a larger dose in a larger patient population is needed to understand the potential of this metabolic intervention. Other promising therapies (minocycline, creatine, and GPI-1485) are also candidates for further testing, having passed the thresholds of futility analyses. However, not meeting futility criteria does not make the eventual finding of efficacy more likely to occur.

One of the biggest challenges ahead is to make sense of the results reported for the three MAO-B inhibitors selegiline, lazabemide, and rasagiline. The clinical improvements seen with each of these compounds suggest a pharmacological class effect. Although each drug shares the property of inhibiting oxidative deamination through MAO type B, it is important to recall that selegiline and rasagiline both possess additional in vitro pharmacological actions that could contribute to neuroprotection. ${ }^{41,46,47}$ The next clinical investigation to report on potential neuroprotection with this class will be the ongoing rasagiline ADAGIO study, which, with 1100 subjects enrolled, will be the largest PD trial to have been conducted with the goal of disease modification.

The legacy of PD neuroprotection studies at this point is a wealth of experience in the challenges and pitfalls of study design and implementation. Data from clinical trials in early PD provide an important resource for studying the natural history of PD and for designing new studies and interpreting the results of others. This information, plus experience in using different clinical rating and neuroimaging study outcome measures, has made it possible for studies to be planned with greater precision as to duration and numbers of subjects required. The limitations of clinical assessment in selecting subjects for enrollment have been highlighted by several studies in which neuroimaging data did not support the diagnostic impressions of enrolling investigators. From this information, future studies can be designed that can model a study cohort with the expectation of less than full clinical diagnostic accuracy.
The lack of efficacy for several compounds with strong hypotheses and promising preclinical profiles emphasizes the limitations of proceeding to clinical trials in the absence of understanding as to the cause or causes of PD. Antioxidants and antiapoptotics might seem to have been eliminated as candidates for a neuroprotective therapy in PD; however, careful attention needs to be given to the details of each study, to learn whether and how experimental plans appropriately tested each concept.

For example, the $\alpha$-tocopherol arm of the DATATOP study investigated a relatively large daily oral intake of this vitamin, but whether this regimen actually increased antioxidant defenses in the brain is not known. ${ }^{105}$ Also in the DATATOP study, the hypothesis for selegiline as an antioxidative therapy was that this drug would inhibit oxyradical production from dopamine turnover, thereby halting disease progression. Subsequent analysis, however, found that this concept was not in fact tested, as shown by measurements of CSF homovanillic acid (HVA). ${ }^{106}$ As the byproduct of dopamine catabolism, HVA formation should be blocked if oxidative deamination had been fully inhibited by selegiline; however, HVA measurements of the selegiline-treated subjects revealed continuing production of HVA, demonstrating that oxidative deamination of dopamine (and hence, hydroxyl radical production) was still occurring. ${ }^{106}$ The investigations using coenzyme $\mathrm{Q}_{10}$ and creatine supplementation also have the challenge of proving adequate drug delivery to the brain to test their experimental concepts.

Several efforts at translating promising candidates (especially animal models of neurotoxin-induced parkinsonism) from laboratory research to clinical trials have failed. In part, this may be due to naïve assumptions in the fragmentary understanding of how PD is initiated and progresses. PD is already an advanced disorder by the time that its clinical features are recognized. The ideal time for a protective intervention already may have passed by many years, or there may be an inherent lifelong risk conferred by genetic or environmental factors that make progressive PD inevitable.

This pessimistic viewpoint should not be the basis for halting further research. Many PD patients would be very satisfied if they could halt the disorder in its mild stages, as is the case for a substantial proportion of patients, who seem to plateau after several years without further worsening. What natural factors are operative in such cases of nonprogressive PD remain to be investigated. Vulnerability of neuronal loss on the basis of genetically determined changes in $\alpha$-synuclein or ubiquitin ligase is under active exploration, and it may be that unraveling their contributions to disease progression might have a therapeutic message for interventions in seemingly sporadic PD. Given the possibility that there is more than one cause, PD may require a multiplicity of treatments. Un- 
fortunately, one implication is that treatments already investigated in clinical trials and rejected as inefficacious might actually have utility for some subcategory of PD.

Until recently, choices for neuroprotective treatments were limited to pharmacological interventions, generally involving small molecules capable of traversing the blood-brain barrier. The advent of recombinant gene therapy has greatly changed the landscape of therapeutic options. ${ }^{107}$ Using viral vectors, it has become possible to equip neurons or other brain cell types with genetic instructions for measured and continuous production of potentially disease-modifying substances. An open-label clinical trial testing a neuroprotective strategy with a gene to synthesize neurturin, an analog of glial-derived neurotrophic factor, is currently underway in 12 subjects. ${ }^{108}$ This work is based in part on the positive results of gene therapy studies using recombinant adeno-associated virus carrying a gene for neurturin in monkeys previously exposed to MPTP. ${ }^{109}$

Other gene therapy strategies have been proposed from preclinical evidence, based on the potentially neuroprotective activity of several biologically generated substances. Like neurturin, erythropoietin generated in situ has been proposed as another means for protection against degenerative changes in PD. ${ }^{110}$ Based on its cytokine properties, erythropoietin can diminish effects of the neurotoxins 6-OHDA and MPTP in rodent experiments (possibly through an anti-inflammatory response against microglial activation). Another proposal from laboratory research comes from a rodent model of parkinsonism in which motor deficits and neuronal dropout were induced by overexpression of $\alpha$-synuclein in dopaminergic SN neurons. ${ }^{111,112}$ In this model, use of recombinant adeno-associated virus to overexpress PARK2 (the parkin gene) greatly attenuated effects of the coexpressed $\alpha$-synuclein gene.

Neuroprotection research for PD is fortunate in that it can borrow concepts from other medical disciplines in the search for promising therapeutic interventions. For example, the oncological drug taxol, ${ }^{113}$ anti-inflammatory medications, ${ }^{114,115}$ and a drug for multiple sclerosis, glatimer, ${ }^{116}$ have each shown therapeutic promise in animal models of parkinsonism. Istradefylline (KW-6002), a xanthine-based antagonist of the adenosine $\mathrm{A}_{2 \mathrm{~A}}$ receptor (and which has symptomatic effects on $\mathrm{PD}$ for parkinsonism), has also been effective for improving recovery in a neurotoxin-induced animal model of parkinsonism. ${ }^{117}$ With an expanding range of therapeutic options, the race for neuroprotection against PD may be in the home stretch.

Dedication: Dedicated to the memory of a colleague and friend, Professor Cliff Shults, M.D., a distinguished Parkinson's disease researcher among whose many research contributions are studies cited in this review.

\section{REFERENCES}

1. Anderson DW, Bradbury KA, Schneider JS. Neuroprotection in Parkinson models varies with toxin administration protocol. Eur J Neurosci 2006;24:3174-3182.

2. Hirsch EC. How to judge animal models of Parkinson's disease in terms of neuroprotection. J Neural Transm Suppl 2006;70:255260.

3. Ravina BM, Fagan SC, Hart RG, et al. Neuroprotective agents for clinical trials in Parkinson's disease: a systematic assessment. Neurology 2003;60:1234-1240.

4. Suchowersky O, Gronseth G, Perlmutter J, Reich S, Zesiewicz T, Weiner WJ. Quality Standards Subcommittee of the American Academy of Neurology. Practice parameter: neuroprotective strategies and alternative therapies for Parkinson disease (an evidence-based review): report of the Quality Standards Subcommittee of the American Academy of Neurology [Erratum in: Neurology. 2006;67:299]. Neurology 2006;66:976-982.

5. Goetz CG, Koller WC, Poewe W, et al. Management of Parkinson's disease: an evidence-based review. Mov Disorders 2002;17 Suppl 4:S1-S166 (abstracts).

6. Goetz CG, Poewe W, Rascol O, Sampaio C. Evidence-based medical review update: pharmacological and surgical treatments of Parkinson's disease: 2001 to 2004. Mov Disorders 2005;20: 523-529.

7. Fearnley J, Lees AJ. Aging and Parkinson's disease: substantia nigra regional selectivity. Brain 1991;114:2283-2301.

8. Parkinson Study Group. DATATOP: a multicenter controlled clinical trial in early Parkinson's disease. Arch Neurol 1989;46: 1052-1060.

9. Parkinson Study Group. Effect of deprenyl on the progression of disability in early Parkinson's disease. N Engl J Med 1989;321: 1364-1371.

10. Parkinson Study Group. Effects of tocopherol and deprenyl on the progression of disability in early Parkinson's disease. N Engl J Med 1993;328:176-183.

11. Shults CW, Haas RH, Beal MF. A possible role of coenzyme Q10 in the etiology and treatment of Parkinson's disease. Biofactors 1999;9:267-272.

12. Tarnopolsky MA, Beal MF. Potential for creatine and other therapies targeting cellular energy dysfunction in neurological disorders. Ann Neurol 2001;49:561-574.

13. Lipton SA, Gu Z, Nakamura T. Inflammatory mediators leading to protein misfolding and uncompetitive/fast off-rate drug therapy for neurodegenerative disorders. Int Rev Neurobiol 2007;82: $1-27$.

14. Deutch AY. Striatal plasticity in parkinsonism: dystrophic changes in medium spiny neurons and progression in Parkinson's disease. J Neural Transm Suppl 2006;70:67-70.

15. Zigmond MJ. Do compensatory processes underlie the preclinical phase of neurodegenerative disease? Insights from an animal model of parkinsonism Neurobiol Dis 1997;4:247-253.

16. Fahn S, Oakes D, Shoulson I, et al.; Parkinson Study Group. Levodopa and the progression of Parkinson's disease. N Engl J Med 2004;351:2498-2508.

17. Fahn S, Elton RL; Members of the UPDRS Development Committee. Unified Parkinson Disease Rating Scale. In: Fahn S, Marsden CD, Calne DB, Goldstein M, eds. Recent developments in Parkinson's disease. Vol. 2. Florham Park, NJ: Macmillan Healthcare Information, 1987:153-164.

18. Shults CW, Oakes D, Kieburtz K, et al.; Parkinson Study Group. Effects of coenzyme Q10 in early Parkinson's disease: evidence for slowing of the functional decline. Arch Neurol 2002;59:15411550 .

19. Guimaraes P, Kieburtz K, Goetz CG, Elm JJ, Palesch YY, Huang P, Ravina B, Tanner CM, Tilley BC. Non-linearity of Parkinson's disease progression: implications for sample size calculations in clinical trials. Clin Trials 2005;2:509-518.

20. Goetz CG, Fahn S, Martinez-Martin P, et al. Movement Disorder Society-sponsored revision of the Unified Parkinson's Disease Rating Scale (MDS-UPDRS): process, format, and clinimetric testing plan. Mov Disord 2007;22:41-47. 
21. Ravina B, Eidelberg D, Ahlskog JE, et al. The role of radiotracer imaging in Parkinson disease. Neurology 2005;64:208-215.

22. Marek K, Jennings D, Seibyl J. Do dopamine agonists or levodopa modify Parkinson's disease progression? Eur J Neurol 2002;9 Suppl 3:15-22.

23. Schillaci O, Pierantozzi M, Filippi L, et al. The effect of levodopa therapy on dopamine transporter SPECT imaging with ${ }^{123}$ I-FPCIT in patients with Parkinson's disease. Eur J Nucl Med Mol Imaging 2005;32:1452-1456.

24. Eckert T, Tang C, Eidelberg D. Assessment of the progression of Parkinson's disease: a metabolic network approach. Lancet Neurol 2007;6:926-932.

25. Huang C, Tang C, Feigin A, et al. Changes in network activity with the progression of Parkinson's disease. Brain 2007;130: $1834-1846$.

26. Elm JJ, Goetz CG, Ravina B, et al. A responsive outcome for Parkinson's disease neuroprotection futility studies. Ann Neurol 2005;57:197-203.

27. Peterson AL, Nutt JG. Treatment of Parkinson's disease with trophic factors. Neurotherapeutics 2008;5:270-280.

28. Spencer JP, Jenner A, Butler J, et al. Evaluation of the prooxidant and antioxidant actions of L-DOPA and dopamine in vitro: implications for Parkinson's disease. Free Radic Res 1996; 24:95-105.

29. Jenner P. Oxidative stress in Parkinson's disease. Ann Neurol 2003;53 Suppl 3:S26-S38.

30. Heikkila RE, Terleckyj I, Sieber BA. Monoamine oxidase and the bioactivation of MPTP and related neurotoxins: relevance to DATATOP. J Neural Transm Suppl 1990;32:217-227.

31. Giladi N, McDermott MP, Fahn S, et al.; the Parkinson Study Group. Freezing of gait in PD: prospective assessment in the DATATOP cohort. Neurology 2001;56:1712-1721.

32. Djaldetti R, Ziv I, Melamed E. The effect of deprenyl washout in patients with long-standing Parkinson's disease. J Neural Transm 2002;109:797-803.

33. LeWitt PA, Oakes D, Cui L; Parkinson Study Group. The need for levodopa as an endpoint of Parkinson's disease progression in a clinical trial of selegiline and $\alpha$-tocopherol. Mov Disord 1997; 12:183-189.

34. Ward CD. Does selegiline delay progression of Parkinson's disease? A critical re-evaluation of the DATATOP study J Neurol Neurosurg Psychiatry 1994;57:217-220.

35. Mäki-Ikola O, Heinonen E. Study design problems of DATATOP study analysis. Ann Neurol 1986;40:946-948.

36. Tetrud JW, Langston JW. The effect of deprenyl (selegiline) on the natural history of Parkinson's disease. Science 1989;245: $519-522$.

37. Myllylä VV, Sotaniemi KA, Vuorinen JA, Heinonen EH. Selegiline as a primary treatment of Parkinson's disease. Acta Neurol Scand Suppl 1991;136:70-72.

38. Pålhagen S, Heinonen EH, Hägglund J, et al.; Swedish Parkinson Study Group. Selegiline delays the onset of disability in de novo parkinsonian patients. Neurology 1998;51:520-525.

39. Pålhagen S, Heinonen E, Hägglund J, et al. Selegiline slows the progression of the symptoms of Parkinson's disease. Neurology 2006;66:1200-1206.

40. Larsen JP, Boas J, Erdal JE; The Norwegian-Danish Study Group. Does selegiline modify the progression of early Parkinson's disease? Results from a five-year study Eur J Neurol 1999; 6:539-547.

41. Tatton W, Chalmers-Redman R, Tatton N. Neuroprotection by deprenyl and other propargylamines: glyceraldehyde-3-phosphate dehydrogenase rather than monoamine oxidase B. J Neural Transm 2003;110:509-515.

42. Parkinson Study Group. A controlled trial of lazabemide (Ro 19-6327) in levodopa-treated Parkinson's disease. Arch Neurol 1994;51:342-347.

43. LeWitt PA, Segel SA, Mistura KL, Schork MA. Symptomatic anti-parkinsonian effects of monoamine oxidase-B inhibition: comparison of selegiline and lazabemide. Clin Neuropharmacol 1993;16:332-337.

44. Parkinson Study Group. Effect of lazabemide on the progression of disability in early Parkinson's disease. Ann Neurol 1996;40:99-107.

45. Akao Y, Youdim MB, Davis BA, Naoi M, Rabey JM. Rasagiline mesylate, a new MAO B inhibitor for the treatment of Parkinson's disease: a double-blind study as adjunctive therapy to levodopa. J Neurochem 2001;78:727-735.

46. Maruyama W, Akao Y, Carrillo MC, et al. Neuroprotection by propargylamines in Parkinson's disease: suppression of apoptosis and induction of prosurvival genes. Neurotoxicol Teratol 2002; 24:675-682.

47. Mandel S, Grunblatt E, Riederer P, et al. Neuroprotective strategies in Parkinson's disease: an update on progress. CNS Drugs 2003;17:729-762.

48. Youdim MB, Amit T, Bar-Am O, Weinstock M, Yogev-Falach M. Amyloid processing and signal transduction properties of anti-Parkinson-anti-Alzheimer neuroprotective drugs rasagiline and TV3326. Ann N Y Acad Sci 2003;993:378-386.

49. Parkinson Study Group. A controlled trial of rasagiline in early Parkinson's disease: the TEMPO Study. Arch Neurol 2002;59: 1937-1943.

50. Carlile GW, Chalmers-Redman RME, Tatton NA, et al. Reduced apoptosis after nerve growth factor and serum withdrawal: conversion of tetrameric gluteraldehyde-3-phosphate dehydrogenase to a dimer. Mol Pharmacol 2000;57:2-12.

51. Stocchi F, Vacca L, Grassini P, et al. Symptom relief in Parkinson disease by safinamide: biochemical and clinical evidence of efficacy beyond MAO-B inhibition. Neurology 2006;67 Suppl 2:S24-S29.

52. Olanow CW, Schapira AH, LeWitt PA, et al. TCH346 as a neuroprotective drug in Parkinson's disease: a double-blind, randomised, controlled trial. Lancet Neurol 2006;5:1013-1020.

53. Parkinson Study Group. A randomized controlled trial comparing pramipexole with levodopa in early Parkinson's disease: design and methods of the CALM-PD Study. Clin Neuropharmacol 2000;23:34-44

54. Parkinson Study Group. Pramipexole vs. levodopa as initial treatment for Parkinson disease. JAMA 2000;284:1931-1938.

55. Parkinson Study Group. Dopamine transporter brain imaging to assess the effects of pramipexole vs levodopa on Parkinson disease progression. JAMA 2002;287:1653-1661.

56. Whone AL, Watts RL, Stoessl AJ, et al.; REAL-PET Study Group. Slower progression of Parkinson's disease with ropinirole versus levodopa: the REAL-PET study. Ann Neurol 2003;54:93101.

57. Oertel WH, Wolters E, Sampaio C, et al. Pergolide versus levodopa monotherapy in early Parkinson's disease patients: the PELMOPET study. Mov Disord 2006;21:343-353.

58. Felten DL, Felten SY, Fuller RW, et al. Chronic dietary pergolide preserves nigrostriatal neuronal integrity in aged Fischer-344 rats. Neurobiol Aging 1992;13:339-351.

59. Ferger B, Teismann P, Mierau J. The dopamine agonist pramipexole scavenges hydroxyl free radicals induced by striatal application of 6-hydroxydopamine in rats: an in vivo microdialysis study. Brain Res 2000;883:216-223.

60. Le WD, Jankovic J. Are dopamine receptor agonists neuroprotective in Parkinson's disease? Drugs Aging 2001;18:389-396.

61. Hall ED, Andrus PK, Oostveen JA, et al. Neuroprotective effects of pramipexole against post-ischemic or methamphetamine-induced degeneration of nigrostriatal neurons. Mov Disord 1996;11 Suppl 1:191 (abstract).

62. Vu TQ, Ling ZD, Ma SY, et al. Pramipexole attenuates the dopaminergic cell loss induced by intraventricular 6-hydroxydopamine. J Neural Transm 2000;107:159-176.

63. Sethi VH, Wu H, Oostveen JA. Neuroprotective effects of the dopamine agonists pramipexole and bromocriptine in 3-acetylpyridine-treated rats. Brain Res 1997;754:181-186.

64. Cassarino DS, Fall CP, Smith TS, Bennett JP Jr. Pramipexole reduces reactive oxygen species production in vivo and in vitro and inhibits the mitochondrial permeability transition produced by the parkinsonian neurotoxin methylpyridinium ion. J Neurochem 1998;71:295-301.

65. Kitamura Y, Kosaka T, Kakimura JI, et al. Protective effects of the antiparkinsonian drugs talipexole and pramipexole against 
1-methyl-4-phenylpyridinium-induced apoptotic death in human neuroblastoma SH-SY5Y cells. Mol Pharmacol 1998;54: $1046-1054$.

66. Zou L, Xu J, Jankovic J, He Y, Appel SH, Le W. Pramipexole inhibits lipid peroxidation and reduces injury in the substantia nigra induced by the dopaminergic neurotoxin 1-methyl-4-phenyl-1,2,3,6-tetrahydropyridine in C57BL/6 mice. Neurosci Lett 2000;281:167-170.

67. Le WD, Jankovic J, Xie W, Appel SH. Antioxidant property of pramipexole independent of dopamine receptor activation in neuroprotection. J Neural Transm 2000;107:1165-1173.

68. Carvey P, Pieri S, Ling Z. Attenuation of levodopa-induced toxicity in mesencephalic cultures by pramipexole. J Neural Transm 1997;104:209-228.

69. Ling ZD, Robie HC, Tong CW, Carvey PM. Both the antioxidant and D3 agonist actions of pramipexole mediate its neuroprotective actions in mesencephalic cultures. J Pharmacol Exp Ther 1999;289:202-210.

70. Anderson DW, Neavin T, Smith JA, Schneider JS. Neuroprotective effects of pramipexole in young and aged MPTP-treated mice. Brain Res 2001;905:44-53.

71. Abramova NA, Cassarino DS, Khan SM, Painter TW, Bennett JP Jr. Inhibition by $\mathrm{R}(+)$ or $\mathrm{S}(-)$ pramipexole of caspase activation and cell death induced by methylpyridinium ion or beta amyloid peptide in SH-SY5Y neuroblastoma. J Neurosci Res 2002;67: 494-500.

72. Takata K, Kitamura Y, Kakimura J, Kohno Y, Taniguchi T. Increase of bcl-2 protein in neuronal dendritic processes of cerebral cortex and hippocampus by the antiparkinsonian drugs, talipexole and pramipexole. Brain Res 2000;872:236-241.

73. Tanaka K, Miyazaki I, Fujita N, Haque ME, Asanuma M, Ogawa $\mathrm{N}$. Molecular mechanism in activation of glutathione system by ropinirole, a selective dopamine D2 agonist. Neurochem Res 2001;26:31-36.

74. Brooks DJ, Frey KA, Marek KL, et al. Assessment of neuroimaging techniques as biomarkers of the progression of Parkinson's disease. Exp Neurol 2003;184 Suppl 1:S68-S79.

75. Rakshi JS, Pavese N, Uema T, et al. A comparison of the progression of early Parkinson's disease in patients started on ropinirole or L-dopa: an ${ }^{18}$ F-dopa PET study. J Neural Transm 2002; 109:1433-1443.

76. LeWitt P, Aizenman E, Newcomer T, Loeffler D; Parkinson Study Group. The search for an endogenous neurotoxin in Parkinson's disease: TOPA and TOPA-quinone. Mov Disord 1994; 9:482 (abstract).

77. Maharaj H, Sukhdev Maharaj D, Scheepers M, Mokokong R, Daya S. L-DOPA administration enhances 6-hydroxydopamine generation. Brain Res 2005;1063:180-186.

78. Olanow CW, Agid Y, Mizuno Y, et al. Levodopa in the treatment of Parkinson's disease: current controversies. Mov Disord 2004; 19:997-1005.

79. Camp DM, Loeffler DA, LeWitt PA. L-DOPA does not enhance hydroxyl radical formation in the nigrostriatal dopamine system of rats with a unilateral 6-hydroxydopamine lesion. J Neurochem 2000;74:1229-1240.

80. Chan PL, Nutt JG, Holford NH. Levodopa slows progression of Parkinson's disease: external validation by clinical trial simulation. Pharm Res 2007;24:791-802.

81. Fahn S. A pilot trial of high-dose $\alpha$-tocopherol and ascorbate in early Parkinson's disease. Ann Neurol 1992;32 Suppl:S128S132.

82. Canet-Avilés RM, Wilson MA, Miller DW, et al. The Parkinson's disease protein DJ-1 is neuroprotective due to cysteine-sulfinic acid-driven mitochondrial localization. Proc Natl Acad Sci U S A 2004;101:9103-9108.

83. The NINDS NET-PD Investigators. A randomized clinical trial of coenzyme Q10 and GPI-1485 in early Parkinson disease. Neurology 2007;68:20-28.

84. Cleren C, Yang L, Lorenzo B, et al. Therapeutic effects of Coenzyme $\mathrm{Q}^{10}\left(\mathrm{CoQ}^{10}\right)$ and reduced $\mathrm{CoQ}^{10}$ in the MPTP model of parkinsonism. J Neurochem 2007 Dec 8 [Epub ahead of print].
85. Matthews RT, Ferrante RJ, Klivenyi P, et al. Creatine and cyclocreatine attenuate MPTP neurotoxicity. Exp Neurol 1999;157: $142-149$.

86. Constantinescu R, McDermott MP, Dicenzo R, et al. A randomized study of the bioavailability of different formulations of coenzyme Q10 (ubiquinone). J Clin Pharmacol 2007;47:15801586.

87. Parkinson Study Group PRECEPT Investigators. Mixed lineage kinase inhibitor CEP-1347 fails to delay disability in early Parkinson's disease. Neurology 2007;69:1480-1490.

88. The NINDS NET-PD Investigators. A randomized, double-blind, futility clinical trial of creatine and minocycline in early Parkinson disease. Neurology 2006;66:664-671.

89. Bender A, Koch W, Elstner M, et al. Creatine supplementation in Parkinson disease: a placebo-controlled randomized pilot trial. Neurology 2006;67:1262-1264.

90. Waldmeier P, Bozyczko-Coyne D, Williams M, Vaught JL. Recent clinical failures in Parkinson's disease with apoptosis inhibitors underline the need for a paradigm shift in drug discovery for neurodegenerative diseases. Biochem Pharmacol 2006;72:11971206.

91. Du Y, Ma Z, Lin S, et al. Minocycline prevents nigrostriatal dopaminergic neurodegeneration in the MPTP model of Parkinson's disease. Proc Natl Acad Sci U S A 2001;98:14669-14674

92. Wu DC, Jackson-Lewis V, Vila M, et al. Blockade of microglial activation is neuroprotective in the 1-methyl-4-phenyl-1,2,3,6 tetrahydropyridine mouse model of Parkinson disease. J Neurosci 2002;22:1763-1771.

93. He Y, Appel S, Le W. Minocycline inhibits microglial activation and protects nigral cells after 6-hydroxydopamine injection into mouse striatum. Brain Res 2001;909:187-193.

94. Diguet E, Fernagut PO, Wei X, et al. Deleterious effects of minocycline in animal models of Parkinson's disease and Huntington's disease. Eur J Neurosci 2004;19:3266-3276.

95. Ishitani R, Kimura M, Sunaga K, Katsube N, Tanaka M, Chuang DM. An antisense oligonucleotide to glyceraldehyde-3-phosphate-dehydrogenase blocks age-induced apoptosis of mature cerebrocortical neurons in culture. J Pharmacol Exp Ther 1996;278: $447-454$.

96. Ishitani R, Sunaga K, Hirano A, Saunders P, Katsube N, Chuang DM. Evidence that glyceraldehyde-3-phosphate-dehydrogenase is involved in age-induced apoptosis in mature cerebellar neurons in culture. J Neurochem 1996;66:928-935.

97. Kragten E, Lalande I, Zimmermann K, et al. Glyceraldehyde-3phosphate dehydrogenase, the putative target of the antiapoptotic compounds CGP 3466 and R-(-)-deprenyl. J Biol Chem 1998; 273:5821-5828.

98. Andringa G, Cools AR. The neuroprotective effects of CGP $3466 \mathrm{~B}$ in the best in vivo model of Parkinson's disease, the bilaterally MPTP-treated rhesus monkey. J Neural Transm Suppl 2000;60:215-225.

99. Maroney AC, Glicksman MA, Basma AN, et al. Motoneuron apoptosis is blocked by CEP-1347 (KT 7515), a novel inhibitor of the JNK signaling pathway. J Neurosci 1998;18:104-111.

100. Parkinson Study Group. The safety and tolerability of a mixed lineage kinase inhibitor (CEP-1347) in PD. Neurology 2004;62: 330-332.

101. Lipton SA, Rosenberg PA. Excitatory amino acids as a final common pathway for neurologic disorders. N Engl J Med 1994; 330:613-622.

102. Dawson TM, Dawson VL. Molecular pathways of neurodegeneration in Parkinson's disease. Science 2003;302:819-822.

103. Rascol O, Olanow CW, Brooks D, et al. A 2-year multicenter placebo-controlled, double blind parallel group study of the effect of riluzole in Parkinson's disease. Mov Disord 2002;17 (Suppl 5):39 (abstract).

104. Jankovic J, Hunter C. A double-blind, placebo-controlled and longitudinal study of riluzole in early Parkinson's disease. Parkinsonism Relat Disord 2002;8:271-276.

105. Vatassery GT, Fahn S, Kuskowski MA; Parkinson Study Group. Alpha tocopherol in CSF of subjects taking high-dose vitamin $\mathrm{E}$ in the DATATOP study. Neurology 1998;50:1900-1902.

106. Parkinson Study Group. Cerebrospinal fluid homovanillic acid in 
the DATATOP study on Parkinson's disease. Arch Neurol 1995;52:237-245.

107. Carlsson T, Björklund T, Kirik D. Restoration of the striatal dopamine synthesis for Parkinson's disease: viral vector-mediated enzyme replacement strategy. Curr Gene Ther 2007;7:109120.

108. Herzog CD, Dass B, Holden JE, et al. Striatal delivery of CERE120 , an AAV2 vector encoding human neurturin, enhances activity of the dopaminergic nigrostriatal system in aged monkeys. Mov Disord 2007;22:1124-1132.

109. Gasmi M, Herzog CD, Brandon EP, et al. Striatal delivery of neurturin by CERE-120, an AAV2 vector for the treatment of dopaminergic neuron degeneration in Parkinson's disease. Mol Ther 2007;15:62-68.

110. Xue YQ, Zhao LR, Guo WP, Duan WM. Intrastriatal administration of erythropoietin protects dopaminergic neurons and improves neurobehavioral outcome in a rat model of Parkinson's disease. Neuroscience 2007;146:1245-1258.

111. Yamada M, Mizuno Y, Mochizuki H. Parkin gene therapy for $\alpha$-synucleinopathy: a rat model of Parkinson's disease [Erratum in: Hum Gene Ther 2005;16:400]. Hum Gene Ther 2005; 16:262-270.

112. Mochizuki H. Gene therapy for Parkinson's disease. Expert Rev Neurother 2007;7:957-960.

113. Ren Y, Liu W, Jiang H, Jiang Q, Feng J. Selective vulnerability of dopaminergic neurons to microtubule depolymerization. J Biol Chem 2005;280:34105-34112.

114. Hunter RL, Dragicevic N, Seifert K, et al. Inflammation induces mitochondrial dysfunction and dopaminergic neurodegeneration in the nigrostriatal system. J Neurochem 2007;100:1375-1386.

115. Chen H, Zhang SM, Hernán MA, et al. Nonsteroidal anti-inflammatory drugs and the risk of Parkinson disease. Arch Neurol 2003;60:1059-1064.

116. Benner EJ, Mosley RL, Destache CJ, et al. Therapeutic immunization protects dopaminergic neurons in a mouse model of Parkinson's disease. Proc Natl Acad Sci U S A 2004;101:9435-9440.

117. Ikeda K, Kurokawa M, Aoyama S, Kuwana Y. Neuroprotection by adenosine $\mathrm{A}_{2 \mathrm{~A}}$ receptor blockade in experimental models of Parkinson's disease. J Neurochem 2002;80:262-270. 